\title{
O USO DA INTERFACE GRÁFICA COMPUTACIONAL COMO FERRAMENTA INTERDISCIPLINAR NA ENGENHARIA
}

Natan Siandas Neves-natansian@ufrj.br

COPPE/UFRJ, Programa de Engenharia Civil, Universidade Federal do Rio de Janeiro Av. Athos da Silveira Ramos, 149, Centro de Tecnologia

21941-909 - Ilha do Fundão - Rio de Janeiro

Resumo: Na engenharia os problemas físicos estão frequentemente associados a fenômenos físicos da natureza governados por modelos matemáticos e princípios físicos. Esse processo de ensino de problemas de engenharia não é um tarefa fácil para os docentes, uma vez que esses problemas requerem usualmente teorias matemáticas abstratas, que podem causar em um primeiro momento aos discentes, uma dificuldade de assimilação do conteúdo. Desta maneira, associado a evolução dos recursos computacionais, o uso de interfaces gráficas educacionais no processo de ensino é uma excelente maneira de direcionar o aprendizado dos discentes. Sendo assim, o presente artigo apresenta um concepção e exemplo inicial acerca do uso de interfaces gráficas aplicada na engenharia. Para tanto, adota-se o problema estrutural de placas delgadas como veículo demonstrativo, apresentando a lógica utilizada, aspectos visuais e resultados que uma interface gráfica pode proporcionar a compreensão do usuário dos fenômenos físicos.

Palavras-chave: Engenharia. Interface gráfica. Aprendizado. Programa computacional.

\section{INTRODUÇÃO}

A aplicação de ferramentas computacionais na seara da ciência e engenharia teve um crescimento visível nas últimas décadas com maior velocidade de processamento de dados, capacidade de armazenamento, redução das dimensões dos componentes digitais, diminuição dos preços, surgimentos de aplicativos e pacotes para aplicações, democratização dos recursos computacionais e dentre outros aspectos (ANDRADE, 2004).

Neste contexto, inúmeras plataformas de implementação computacional foram difundidas e ganharam espaço em aplicações acadêmicas voltadas o ensino-aprendizado dos discentes. Atualmente, alguns recursos tornaram-se populares em salas de aulas e pesquisas cientificas, auxiliando os docentes na transmissão de conteúdo e aos discentes no desenvolvimento de ferramentas autorais de análise sem necessidade de ter um conhecimento aprofundado em programação computacional (CARVALHO et al., 2008). Destaca-se, por exemplo, alguns softwares que apresentam uma lógica simples e direta na programação, como Matlab, Lua, Python, Octave e Scilab. Essas ferramentas permitem o desenvolvimento rápido, intuitivo e prático de interfaces gráficas de usuário (GUI - Graphical User Interface). As interfaces são elementos essenciais para simplificação e tradução didática do problema ao usuário, não apresentando extensas linhas de códigos.

Em específico na área da engenharia, esses recursos computacionais são importantes devido ao grande volume de modelos matemáticos abstratos, exigindo um auxílio de ferramentas na compreensão dos problemas. Dessa maneira, a utilização dessas novas metodologias didáticas baseadas em programas com aplicação em problemas físicos de engenharia auxiliam os docentes na transmissão do conteúdo, uma vez que essas ferramentas 
permitem ao usuário adquirir características como pesquisar científica, multidisciplinaridade, associação física-matemática e simulação de efeitos e parâmetros físicos associados aos problema estudados, e não simplesmente gerar resultados aleatórios e ver gráficos coloridos sem um real compreensão física atrelado ao comportamento do fenômeno (PIMENTEL, 2017).

\section{FUNDAMENTOS TEÓRICOS DA MODELAGEM DE PLACAS DELGADAS}

Na construção de uma interface gráfica computacional é necessário ter conhecimentos prévios de fundamentos teóricos clássicos associados ao problema físico estudado, a fim de compreender os efeitos, comportamentos físicos, grandezas e variáveis matemáticas associadas ao fenômeno. Em particular para engenharia estrutural, disciplinas como resistência dos materiais, mecânica estática e dinâmica, análise estrutural, elasticidade, dimensionamento e projeto de elementos estruturais de concreto armado, madeira, aço ou mistas, são disciplinas curriculares usuais em cursos de graduação ou pós-graduação de engenharia, essenciais para formação da base conceitual e física dos discentes. Nota-se que a ausente desses conceitos indica um déficit físico na elaboração de interface gráfica, uma vez que se perde informação sobre o problema ou possíveis erros na implementação dos códigos por falta de conhecimento físico (NEVES, 2019; SHIOTANI; SOUZA, 2020).

Neste contexto, uma vez que a interface gráfica escolhida para exemplificação no presente trabalho é na seara da teoria de placas delgadas. Apresentam-se nesta seção as principais hipóteses, conceitos e equacionamento sobre esse campo. Primeiramente, ressaltase que a teoria de placas é uma aproximação de engenharia que reduz o problema tridimensional original a um problema bidimensional mais simples. Para muitas aplicações de engenharia limitada ao caso de placas delgadas, felizmente, essa teoria fornece soluções realistas e traduzem satisfatoriamente o comportamento das estruturas.

Em relação às hipóteses simplificadoras de cálculo, considera-se que material é homogêneo, isotrópico, elástico, linear fisicamente e os deslocamentos são pequenos. Em adicional, adota-se que a superfície média não se deforma durante a flexão, despreza-se os efeitos de cisalhamento e as seções transversais permanecem normais à superfície plana (SZILARD, 2004).

Figura 1 - Superfície (ou plano) média da placa (esquerda) e forças verticais de cisalhamento e momentos atuando em um elemento infinitesimal de placa sujeito à ação de uma força distribuída na superfície (direita).
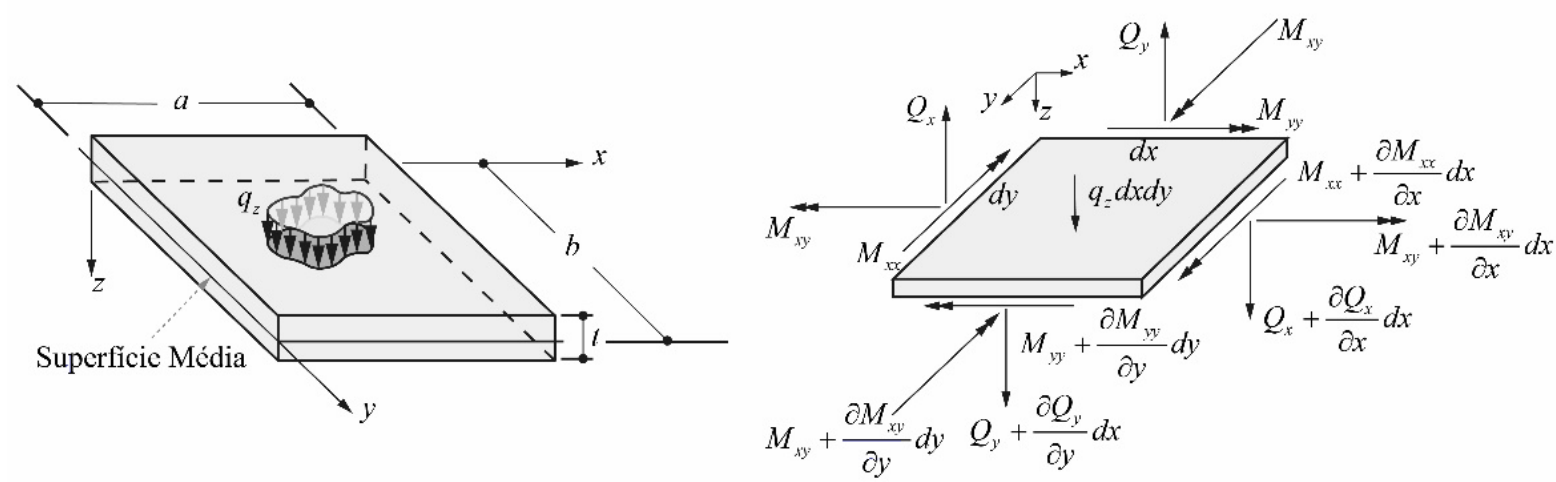

Fonte: Acervo Pessoal.

A modelagem de placas é desenvolvida em torno de uma superfície média e o carregamento é normal ao plano, conforme esquematizado na Figura 1. Assume que as placas 
delgadas (ou finas) são caracterizadas por terem sua espessura $t \ll \ell$, sendo $\ell$ uma dimensão típica da placa, ou seja, a espessura da placa é pequena em relação às outras dimensões.

Além disso, deve-se compreender que grande parte dos fenômenos físicos presentes na natureza são modelados por equações diferenciais (ordinárias ou parciais). O problema de placa fina não é diferente, realizando o equilíbrio de forças e momentos em um elemento infinitesimal (Figura 1), tem-se um conjunto de expressões diferenciais em função dos esforços internos, conforme mostra a seguir:

$$
\begin{gathered}
\frac{\partial Q_{x}}{\partial x}+\frac{\partial Q_{y}}{\partial y}+q_{z}=0 \\
\frac{\partial M_{x y}}{\partial x}-\frac{\partial M_{y}}{\partial y}+Q_{y}=0 \\
\frac{\partial M_{x y}}{\partial y}-\frac{\partial M_{x}}{\partial x}+Q_{x}=0
\end{gathered}
$$

Baseado nas Equações (1), (2) e (3), e realizando algumas operações algébricas pode-se chegar na equação diferencial de governo baseada na teoria de placa de Kirchhoff representativa da deflexão da placa, w, conforme exibe na Equação (4).

$$
\frac{\partial^{4} w}{\partial x^{4}}+2 \frac{\partial^{4} w}{\partial x^{2} \partial y^{2}}+\frac{\partial^{4} w}{\partial y^{4}}=\nabla^{2}\left(\nabla^{2} w\right)=\frac{q_{z}}{D}
$$

Onde $q_{z}$ é o carregamento uniformemente distribuída na placa e $D$ é conhecido como rigidez à flexão da placa igual a $E t^{3} / 12\left(1-v^{2}\right)$, em que $E$ é o módulo de elasticidade, $t$ é a espessura e $v$ é o coeficiente de Poisson. Além disso, nota-se que a equação de governo é caracterizada pela presença do operador biharmônico.

Em paralelo, devem-se impor condições de contorno ao problema, semelhante às ideias e considerações da teoria de vigas de Euler-Bernoulli, contudo, na teoria de placas aplicadas em um contexto bidimensional. Por exemplo, para uma borda $x=i$, simplesmente apoiada, a deflexão ao longo da borda apoiada deve ser nula. Ao mesmo tempo em que se permite a rotação na borda, não existe momento fletor. Matematicamente, escrevem-se as seguintes sentenças:

$$
[w]_{x=i}=0 \quad\left[\frac{\partial^{2} w}{\partial x^{2}}+v \frac{\partial^{2} w}{\partial y^{2}}\right]_{x=i}=0
$$

Dentre as propostas de solução analítica para teoria de placas finas, considere a solução por séries trigonométricas proposta por Navier para placa totalmente apoiada sujeita a uma carga uniformemente distribuída, $q_{0}$, conforme posto na Equação (6) (TIMOSHENKO; WOINOWSKY-KRIEGER, 1959).

$$
w=\frac{16 q_{0}}{\pi^{6} D} \sum_{m=1}^{\infty} \sum_{n=1}^{\infty} \frac{\sin \left(\frac{m \pi x}{a}\right) \sin \left(\frac{m \pi x}{b}\right)}{m n\left(\frac{m^{2}}{a^{2}}+\frac{n^{2}}{b^{2}}\right)^{2}}
$$

A flecha máxima para uma placa quadrada de lado $a$ ocorre no meio vão da placa, igual a $0,00416 q_{0} a^{4} / D$. Com base na Equação (6) é possível obter variáveis secundárias importantes 
para análise estrutural. As reações de apoio, $V_{1 x}$, na borda $x=0$ é constituída por duas contribuições e em função do deslocamento, conforme mostrado a seguir.

$$
\left.V_{1 x}\right|_{x=0}=\left.\left[Q_{1 x}+\frac{\partial T_{1 x y}}{\partial y}\right]\right|_{x=0}
$$

Onde:

$$
Q_{1 x}=D\left[\frac{\partial^{3} w}{\partial x^{3}}+\frac{\partial^{3} w}{\partial x \partial y^{2}}\right] \quad T_{1 x y}=D(1-v) \frac{\partial^{2} w}{\partial x \partial y}
$$

Além disso, têm-se ainda as reações concentradas nos cantos da placa retangular, dada pela Equação (9).

$$
R=2 D(1-v) \frac{\partial^{2} w}{\partial x \partial y}
$$

Convém frisar que a solução de Navier caracterizadas por processos matemáticos baseados em séries duplas trigonométricas permite descrever o carregamento externo em função de séries também. Desta maneira, aplicando esses conceitos escreve-se a sentença a seguir:

$$
f(x, y)=\sum_{m=1}^{\infty} \sum_{n=1}^{\infty} a_{m n} \sin \left(\frac{m \pi x}{a}\right) \sin \left(\frac{n \pi y}{b}\right)
$$

Para o caso da carga uniformemente distribuída na superfície da placa fina retangular, o coeficiente $a_{m n}$ é igual a $16 q_{0} / \pi^{2} m n$. Para detalhes da modelagem e outras soluções de casos com vinculações e carregamentos diferentes vide Timoshenko e Woinowsky-Krieger (1959).

Em adicional, as soluções analíticas da equação diferencial biharmônica são disponíveis para casos simples. Usualmente, para generalizar as análises, aplicam-se procedimentos aproximativos para solução, como elementos finitos, diferenças finitas ou elementos de contorno, conforme pode ser visto em detalhes nas obras de Neves et al. (2018) e Pinheiro et al. (2017).

\section{INTERFACE GRÁFICA PARA ENGENHARIA}

Os problemas físicos de engenharia são modelados frequentemente por equações matemáticas que buscam aproximar o comportamento real do fenômeno. Essas equações diferenciais apresentadas anteriormente carregam entre si um forte aspecto físico do problema estudado. Todavia, em muitas circunstancias, essa modelagem matemática abstrata causa dificuldade no entendimento e visualização do problema para os discentes. Sendo assim, para motivação e aprofundamento teórico do fenômeno físico, o uso de aspectos gráficos didáticos auxiliam no ganho de sensibilidade acerca do comportamento do problema (NEVES et al., 2019). 
Figura 2 - Lógica de programação e aspectos visuais gerais da interface gráfica construída para análise pedagógica de placas delgadas.
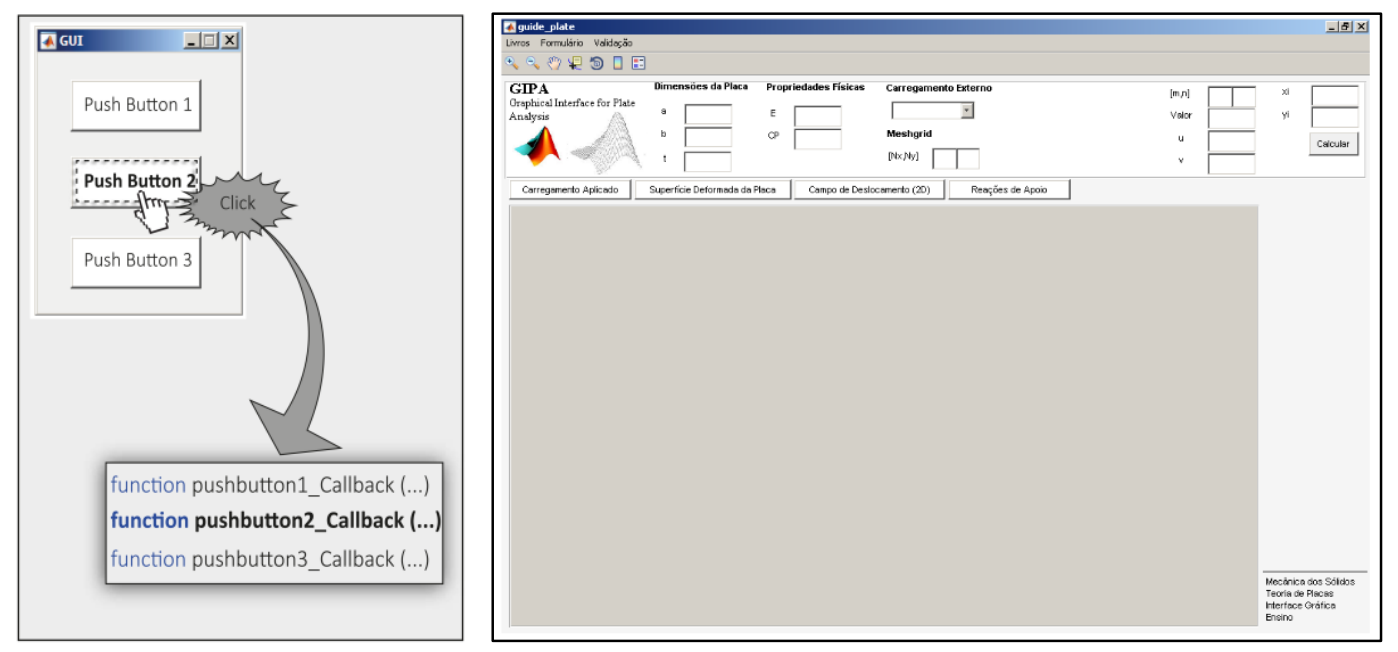

Fonte: Acervo Pessoal.

Neste contexto, baseado na teoria de placas finas, neste trabalho elaborou uma interface gráfica puramente analítica para estudo de placas finas retangulares simplesmente apoiadas sob ação de carregamentos externos, denominada de GIPA (Graphic Interface for Plate Analysis) e desenvolvido em ambiente MATLAB R2015a, conforme mostrado na Figura 2. A lógica de implementação utilizada para elaboração da interface é baseada em uma programação de eventos, ou seja, para cada ação diferente do usuário aplicada em elemento gráfico (botoes, slider, menus e dentro outros) tem-se associado uma função denominada de Callback (RANGEL; MARTHA, 2018). Dentro de cada função são programados os comados e fórmulas matemáticas a serem executadas quando forem acionados pelo usuário.

$\mathrm{Na}$ parte superior da Figura 2 apresenta-se os parâmetros iniciais para a simulação, contemplando as dimensões da placa $a, b$ e $t$, e as propriedades do material. Além disso, pode-se escolher os tipos de carregamentos externos aplicados na superfície da placa, como uniformemente distribuída, concentrado, parcialmente distribuída em uma região e senoidal, conforme esquematizado na Figura 3.

Figura 3 - Tipos de carregamentos externos disponíveis na interface gráfica.

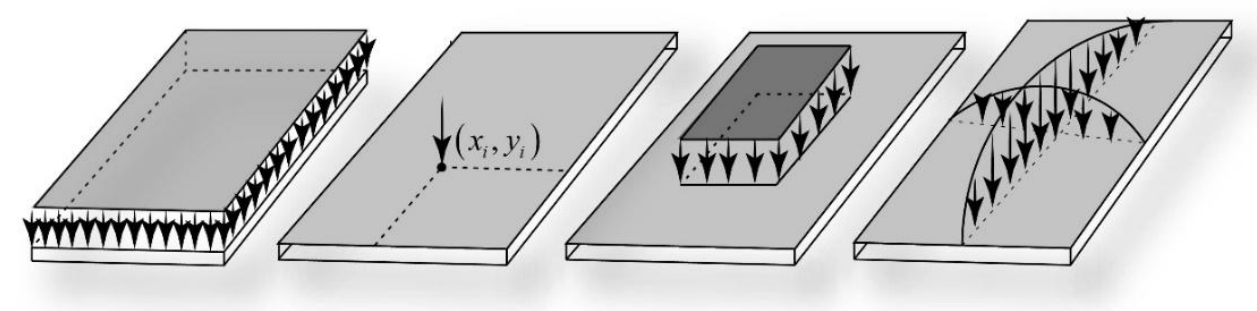

Fonte: Acervo Pessoal.

A interface computacional construída é baseada na solução por séries trigonométricas, logo, solicita-se ao usuário a inserção do número de termos da série, indicados por $n$ e $m$. Outro ponto de destaque é o meshgrid, onde são construídos os malha de pontos para as bases de cálculo das variáveis do problema, o usuário específica o número de pontos na direção $x \mathrm{e}$ $y$. Em termos de avaliação de resultados, a GIPA possibilidade visualizar graficamente alguns parâmetros importantes para análise de placas, como aproximação por série do carregamento 
(C) COBENGE

"Os desafios para formar hoje o engenheiro do amanhã"
O1 a 03 de dezembro Evento On-line

aplicado, vista da superfície deformada da placa (3D) e do campo de deslocamento bidimensional (2D), e as reações de apoio distribuída ao longo das bordas e concentradas nos cantos da placa.

Figura 4 - Aproximação do carregamento externo para 5 e 50 termos na série.
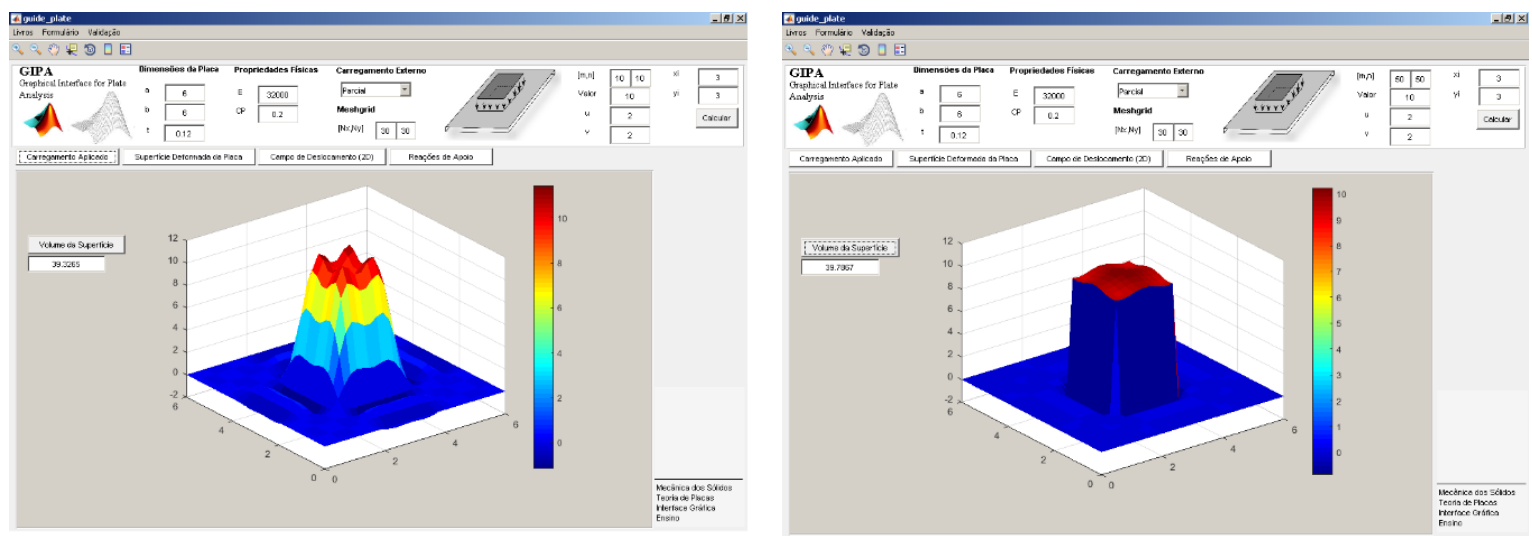

Fonte: Acervo Pessoal.

Para apresentar os resultados, considere um caso teste de uma placa delgada quadrada de dimensão igual a $6 \mathrm{~m}$ e espessura de $0,12 \mathrm{~m}$, o módulo de elasticidade é $32 \mathrm{MPa}$ e o coeficiente de Poisson igual a 0,2, além disso, adota-se como exemplo o carregamento parcialmente distribuído. Na Figura 4 mostra-se como o carregamento externo é aproximado pela série trigonométrica, considerando 5 e 50 termos. Além disso, nesse aba da interface é possível visualizar o volume do gráfico tridimensional, representativo da intensidade da carga aplicada.

$\mathrm{Na}$ segunda e terceira aba de resultados tem-se a representação do campo de deslocamento tridimensional e bidimensional, conforme apresentado na Figura 5. Além disso, é possível obter o valor da flecha máxima da placa na opção ao lado do gráfico. Essas visualizações são importantes para engenharia a fim de verificar as regiões críticas na placa para realização de projetos e análises.

Figura 5 - Campo de deslocamento 3D e 2D.

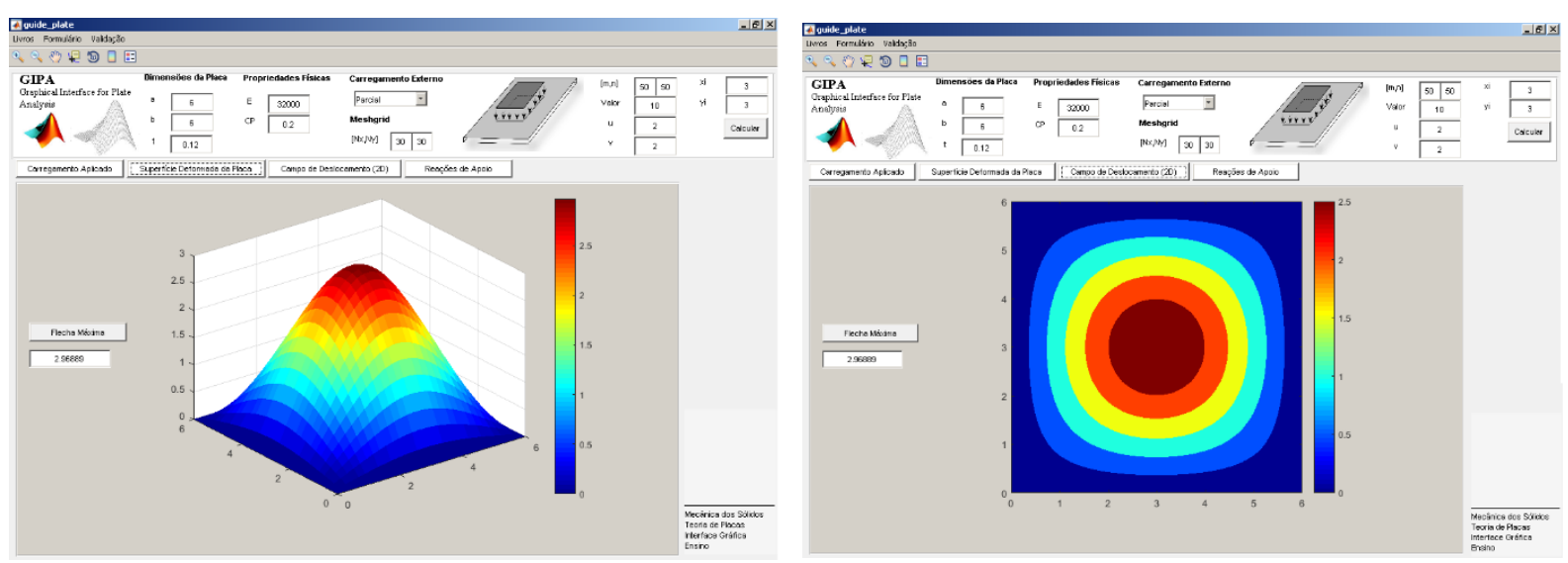

Fonte: Acervo Pessoal. 
(C) COBENGE

"Os desafios para formar hoje o engenheiro do amanhã"

Em adicional, a última aba da interface gráfica é relativa as reações de apoio distribuídas ao longo das bordas e concentradas nos cantos da placa retangular, conforme mostra a Figura 6. Diferentemente em problemas unidimensionais da análise estrutural de vigas reticuladas, onde se tem reações concentradas em apoios, em placas tem-se reações distribuídas nos apoios.

Figura 6 - Reações distribuídas e concentradas na placa.

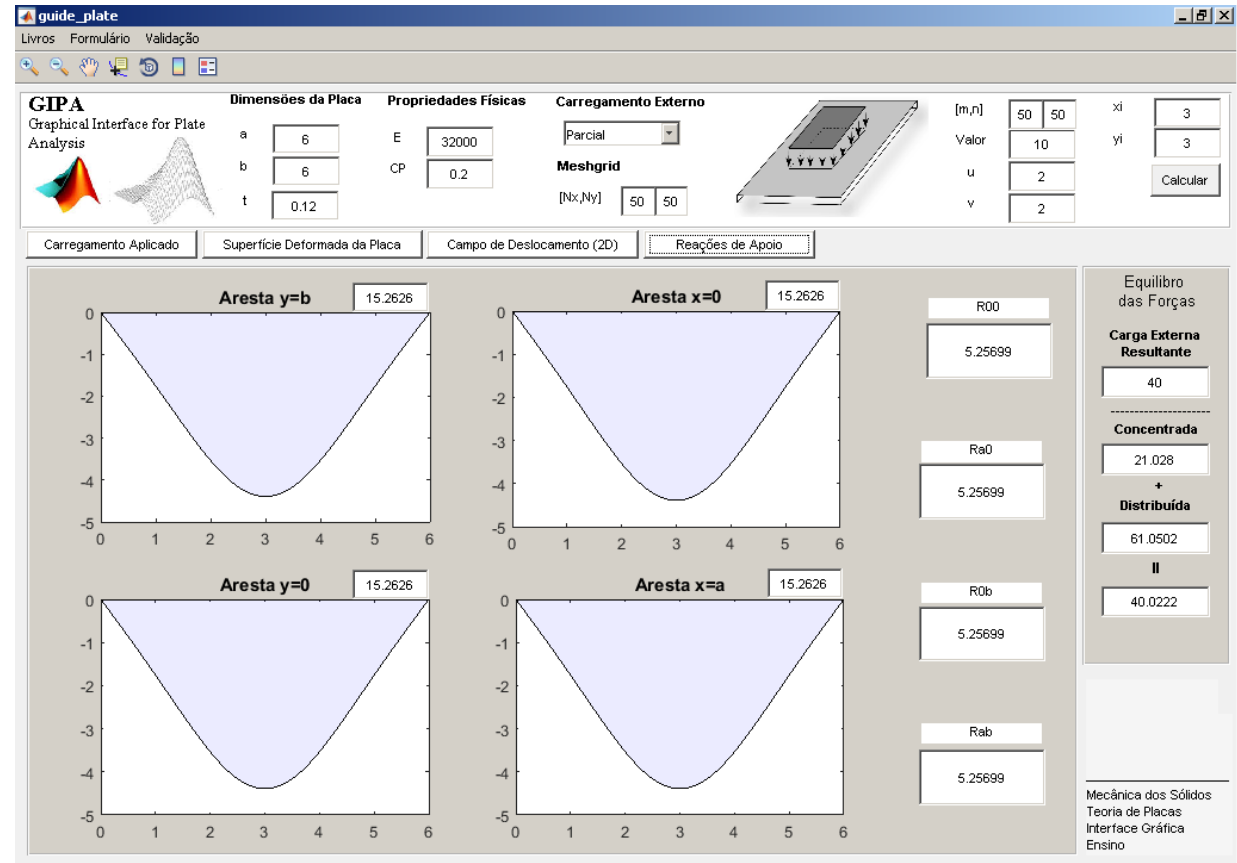

Fonte: Acervo Pessoal.

Outro ponto de destaque é o equilíbrio das forças, representado ao lado direito na interface, onde observa-se que a força resultante proveniente do carregamento externo deve ser igual a soma das contribuições das forças concentradas nos cantos e distribuídas ao longo das bordas. Todavia, esse resultado variável com a quantidade de pontos na malha de cálculo e o número de termos na série dupla trigonométrica escolhida pelo usuário. Desta forma, na Figura 6 observa-se uma diferença entre os valores finais obtidos, onde a carga externa resultante chega ao valor de $40 \mathrm{kN}$ e a somatória das forças internas com valor de 40,0222 $\mathrm{kN}$.

\section{ASPECTOS RELACIONADOS AO ENSINO-APRENDIZAGEM}

O uso de interfaces gráficas no processo pedagógico de ensino de discentes em cursos de engenharia torna-se um veículo complementar que atua como objetivo facilitador do conhecimento. A possibilidade que uma ferramenta dessa natureza dá ao aluno em relação à liberdade de realizar experimentações e testes com diferentes situações físicas e parâmetros relacionados ao problema estudado. Além disso, permite ao aluno uma associação multidisciplinar no aprendizado durante a construção e execução desses aplicativos educacionais.

Por exemplo, no caso da interface gráfica no contexto da teoria de placas delgadas apresentado no item anterior do presente trabalho, pode-se observar a conectividade entre os conceitos do cálculo como série trigonométricas, equações diferenciais, derivadas e integrais, 
além dos recursos gráficos relacionados aos algoritmos computacionais, bem como as disciplinas relacionados ao comportamento das estruturas, como estruturas de concreto (dimensionamento de lajes de concreto, onde tem-se as tabelas de cálculo e métodos simplificados que em muitas cenários são provenientes dos resultados dessas teorias clássicas), análise estrutural ou mecânica geral, com os conceitos de esforços internos e deslocamentos. Ou seja, essas ferramentas realizam uma verdadeira mistura de conhecimento de disciplinas cursadas ao longo dos cursos de graduação ou pós-graduação de engenharia, realizando uma construção step-by-step dos conceitos físicos.

Outro ponto importante é o uso dessas ferramentas como apoio em disciplinas ministradas pelos docentes, uma vez que se pode criar um interesse e motivação por parte dos alunos quando expostos a essas ferramentas em sala de aula, fortalecendo a interação entre discente-docente. Alguns conceitos físicos-matemáticos são demasiados abstratos no processo de transmissão do conteúdo aos alunos, contudo, pode-se alcançar com o uso das interfaces gráficas novos recursos facilitadores para compressão dos alunos desses conceitos, que não seriam tangíveis com livros e metodologias clássicas de ensino. Por exemplo, as reações de apoio distribuídas ao longo das bordas e concentradas nos cantos das placas são ideias não triviais ao primeiro momento que se estuda esse conteúdo, com auxílio de uma ferramenta computacional facilita o entendimento desses conceitos e como se comportamento essas variáveis dependendo dos parâmetros físicos adotados na simulação. Outro exemplo associado aos aspectos da matemática abstrata é a aproximação do carregamento da placa por séries trigonométricas, conforme mostrado na Equação (10). Por exemplo, como se descreve um carregamento concentrado de uma placa por séries? Essa é pergunta que pode ser resolvido facilmente com auxílio de recursos gráficos. Desta forma, o usuário aprenderá que para determinadas dimensões e carregamentos, tem-se um comportamento específico associado.

Finalmente, essas ferramentas gráficas educacionais, dependendo da natureza do problema, podem ser utilizadas para conferência de cálculos, servindo como apoio aos docentes na elaboração de exercícios e também aos estudos de fixação dos discentes. Sendo assim, o usuário pode solucionar alguns exercícios manualmente, e posteriormente realizar a avaliação dos resultados obtidos com as ferramentas rapidamente.

\section{CONSIDERAÇÕES FINAIS}

Neste trabalho apresentou uma interface gráfica desenvolvida para análise de placas delgadas retangulares simplesmente apoiada submetida a carregamentos externos. Visando mostrar a importância dessas ferramentas na correlação entre o aprendizado dos discente e a modelagem matemática da engenharia.

Esses recursos gráficos simples e específicas a um certo problema físico são interessante e eficientes para os discentes em relação a exploração e experimentação de parâmetros preliminares do fenômeno. Essa interface desenvolvida para análise de placas é um exemplo da versatilidade e potencial dessa ferramenta para ensino e dinamismo em sala de aula, podendo ser aplicada em inúmeras área de pesquisas, transitando da área estrutural, solos, matemática, física, fluidos, eletromagnetismo, química e dentre outras.

Portanto, as interfaces gráficas permitem uma interação multidisciplinar para os alunos, possibilitando aos discentes uma motivação em todo processo de ensino de engenharia, uma vez que as ferramentas gráficas são essenciais para representação e análise de projetos, como também um potencial crítico de assimilação e apresentação dos conteúdos 


\section{REFERÊNCIAS}

ANDRADE, Cid M. G. Software Livre: Alguns Aplicativos Científicos para Engenharia. In: COBENGE-Congresso Brasileiro de Ensino de Engenharia, 2004, Brasília. Anais. 2004.

CARVALHO, Carlos Vitor Alencar; CARVALHO, Janaina Veiga; SILVA, Júlio César. VAPD-2D: Simulador para Apoio ao Ensino de Engenharia Ambiental. Revista Eletrônica TECCEN, v. 1, n. 2, p. 01-08, 2008.

MATLAB, Version. 8.5. 0.197613 (R2015a). The MathWorks Inc., Massachusetts, 2015.

NEVES, Natan Sian. Modelo computacional avançado para análise de estruturas sob ação de gradientes térmicos. Dissertação (Mestrado) — Universidade Federal do Espírito Santo, Vitória, 2019.

NEVES, Natan Sian; PINHEIRO, Vitor Pancieri; CAMARGO, Rodrigo Silveira.; Desenvolvimento de uma interface gráfica educacional para ensino de elementos finitos aplicado a problemas de viga sob base elástica, p. 67-70 . In: X Encontro Científico de Física Aplicada, 2019, Vitória. Anais. ES, 2019.

NEVES, Natan Sian; SOUZA, Rovena Meirelles; PINHEIRO, Vitor Pancieri. A importância dos modelos teóricos e técnicas numéricas na construção de um elo com a prática da engenharia estrutural. Revista eixo, v. 7, n. 1, p. 26-35, 2018.

PIMENTEL, Flaider Alves. Desenvolvimento de interface gráfica e cálculo de vigas hiperestáticas aplicados ao ensino de Engenharia. RCT-Revista de Ciência e Tecnologia, v. 3, n. 5, 2017.

PINHEIRO, Vitor Pancieri; LOEFFLER, Carlos Friedrich; NEVES, Natan Sian; CANDIDO, Daniel Carvalho de Moura. Analysis of relative performance among numerical methods applied to the thin plate theory. In. CILAMCE - Proceedings of the XXXVIII Iberian LatinAmerican Congress on Computational Methods in Engineering, 2017, Florianopolis. Anais, SC, 2017

RANGEL, Rafael Lopez; MARTHA, Luiz Fernando. LESM-An object-oriented MATLAB program for structural analysis of linear element models. Computer Applications in Engineering Education, v. 27, n. 3, p. 553-571, 2019.

SHIOTANI, André Kazuhiro; SOUZA, Luiz Antonio Farani. Desenvolvimento de interface gráfica de usuário no programa scilab para análise não linear física de barra. Revista Tecnológica, v. 29, n. 2, p. 541-552, 2020.

SZILARD, R. Theories and applications of plate analysis. New Jersey: John Willey \& Sons, 2004.

TIMOSHENKO, Stephen P.; WOINOWSKY-KRIEGER, Sergius. Theory of plates and shells. McGraw-hill, 1959. 


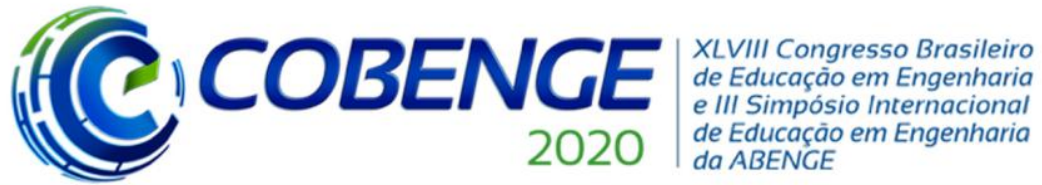

"Os desafios para formar hoje o engenheiro do amanhã"

\title{
THE USE COMPUTACIONAL GRAPHICS INTERFACE AS AN INTERDISCIPLINARY TOOL IN ENGINEERING
}

\begin{abstract}
In engineering, physical problems are often associated with physical phenomena of nature governed by mathematical models and physical principles. This process of teaching engineering problems is not an easy task for teachers, since these problems usually require abstract mathematical theories, which can generate difficulty in assimilating the content. In this way, associated with the evolution of computational resources, the use of graphic educational interfaces in the teaching process is an excellent way to direct students' learning. Therefore, this article presents an initial concept and example about the use of graphical interfaces applied in engineering. For that, the structural problem of thin plates is adopted as a demonstrative vehicle, presenting the logic used, visual aspects and results that a graphic interface can provide the user's understanding of physical phenomena.
\end{abstract}

Keywords: Engineering, Graphical interface, Learning, Computer program 